\title{
Flutter ablation with remote magnetic navigation: comparison between the 8-mm tip, the irrigated tip and a manual approach
}

\author{
Wim ANNÉ, MD, PhD; Bruno SCHWAGTEN, MD; Petter JANSE, RN; Tamas BAUERNFEIND, MD; \\ Yves VAN BELLE, MD, PhD; Natasja DE GROOT, MD, PhD; Paul KNOPS, BSc; Luc JORDAENS*, MD, PhD; \\ Tamas SZILI-TOROK ${ }^{\star}, \mathrm{MD}, \mathrm{PhD}$ \\ Department of Cardiology; Thoraxcentre, Erasmus MC, Rotterdam, the Netherlands.
}

Introduction Remote magnetic navigated ablation has proven its feasibility in a large group of arrhythmias. Until now only scarce data are available on the use for atrial flutter. In this study we compared remote magnetic navigation (RMN), using non-irrigated and irrigated tip catheters, to manual radiofrequency ablation for ablating typical atrial flutter.

Methods The 3 study groups consisted of 17 patients treated with RMN 8-mm tip; 14 patients with RMN irrigated tip; and 24 patients with a manual 8-mm tip. The primary outcome was the number of patients in whom bidirectional isthmus block could be obtained with $\leq 15$ applications. Secondary end points were the median number of applications needed, the need to switch to a manual irrigated tip catheter, the procedural and fluoroscopy times.

Results There was no significant difference in the primary end point (RMN 8 mm-tip group: 59\%, RMN irrigated tip group: $64 \%$ and manual group: $83 \%$ ). The median number of applications needed to obtain block was higher in the RMN groups compared to the manual group. In 5 patients from the RMN 8-mm tip group, 1 in the RMN irrigated tip group and 1 in the manual group, a switch to a manually irrigated tip catheter was performed. There was no difference in fluoroscopy time, but procedural time was significantly longer in the RMN groups compared to the manual group ( $P=0.03$ ).

Conclusions The use of magnetic navigation for the ablation of atrial flutter is feasible but not superior to a manual approach. There was no difference concerning the primary end point of acute success within 15 applications. Overall, more applications were needed and procedure times were longer with RMN but RMN with the irrigating tip is promising.

Keywords Atrial flutter-remote magnetic navigation-radiofrequency ablation-cavotricuspid isthmus.

\section{INTRODUCTION}

Radiofrequency catheter ablation of the isthmus between the tricuspid annulus and the inferior vena cava has become a first-line treatment for patients suffering from recurrent atrial flutter ${ }^{1}$. Since the introduction of the concept of bidirectional block over the isthmus, this method has evolved into a highly effective procedure.

*Luc Jordaens and Tamas Szili-Torok share the senior authorship Address for correspondence:

Wim Anné MD, PhD, Thoraxcenter, Erasmus MC, PO Box 2040, 3000 CA Rotterdam, The Netherlands. E-mail:wim.anne1@gmail.com Received 31 December 2010, accepted for publication 6 January 2011.
New treatment strategies are being developed with as major goals to further improve efficiency, efficacy and safety of this procedure.

Remote magnetic navigation (RMN) has proven its feasibility in a large group of arrhythmias such as atrial fibrillation ${ }^{2}$, AV-node re-entry tachycardia ${ }^{3}$, congenital heart disease ${ }^{4}$ and ventricular tachycardia ${ }^{5}$. The advantages of RMN are the very precise and accurate navigation possibilities and the reduced radiation exposure for the operator. The soft catheter makes cardiac perforation unlikely. The most often used catheter types, the $8-\mathrm{mm}$ tip and the irrigated tip, are also available for RMN. Recently, Vollmann et al. reported longer procedural and ablation times with the 8-mm RMN guided catheter compared to a manual approach ${ }^{6}$. There has been much hope that these results could be improved with the use of an irrigated RMN-guided catheter. However, until 
now, no comparison between the irrigated-tip RMN guided catheter, the $8-\mathrm{mm}$ RMN guided catheter and a manual approach has been made.

Nowadays, different strategies to ablate the cavotricuspid isthmus exist: some prefer to drag the ablation catheter while ablating; others use a point-by-point approach. Recently a third method has been described guided by the maximum voltage over the isthmus, based on the pathological observation that the cavotricuspid isthmus is composed of distinct muscular bundles, responsible for the electrical conduction ${ }^{7}$. The maximum voltage technique aims to target only these muscular electrical conducting - bundles and not the interlaying - non-conducting - connective tissue. This is achieved by sequentially ablating the highest voltages on the isthmus till bidirectional block is reached. This technique was assessed during coronary sinus pacing, sinus rhythm and during atrial flutter and proven to be a feasible $\operatorname{method}^{8}$. This technique reduced the number of ablation lesions, the fluoroscopy and the procedure times needed to achieve bidirectional isthmus block.

The primary aim of this project was to assess the feasibility and efficacy of the use of RMN (Stereotaxis, Niobe) in conjunction with the maximum voltage technique for ablating the cavotricuspid isthmus. We compared both the $8-\mathrm{mm}$ remote magnetic navigated catheter, the irrigated remote magnetic navigated catheter with a manual approach using an 8 -mm tip catheter.

\section{METHODS}

\section{Patient characteristics}

Between May 2007 and March 2010, 55 consecutive patients were included in this single-centre registry. Only patients with a first procedure were included. We excluded patients with congenital heart disease and heart transplantation. In total, 31 patients were treated with the aid of RMN: 17 with an 8-mm tip catheter, and 14 patients with an irrigated tip. The other 24 patients were treated with a manual $8-\mathrm{mm}$ tip catheter. The choice whether a patient was treated with RMN or with a manual approach was based on the availability of the RMN room. We started the RMN irrigated tip instead of the RMN 8-mm catheter from November 2008 on.

\section{Ablation procedure}

The procedures were performed in the fasting state, under local anaesthesia and light sedation with diazepam. Analgesia was given with i.v. fentanyl. A 20-pole catheter (Inquiry H, Boston Scientific) was advanced into the right atrium and positioned around the tricuspid annulus; a decapolar catheter (Polaris, Boston Scientific) was positioned in the coronary sinus. All patients were ablated while pacing the os of the coronary sinus.

All procedures were carried out by a junior and senior electrophysiologist.

Procedural times were measured from the puncture till 30 minutes after the last applications.

\section{Manual group}

Ablation in the conventional group was performed with an 8-mm Blazer catheter (Boston Scientific, Inc.). The isthmus between the tricuspid valve and the vena cava inferior was carefully mapped and the highest peakto-peak bipolar atrial electrograms were searched for. Ablation was first directed to the highest potential. If the ablation lesion did not result in bidirectional block, the next largest atrial electrogram was subsequently targeted for ablation until bidirectional block was present. Catheter settings were $60 \mathrm{~s}, 60 \mathrm{~W}, 60^{\circ} \mathrm{C}$. We used standard pacing manoeuvres to verify bidirectional block. If bidirectional block could not be achieved within 15 applications a switch to a cooled-tip catheter was allowed (Thermocool, Biosense Webster, settings: $60 \mathrm{~s}$, $35 \mathrm{~W}, 48^{\circ} \mathrm{C}$, cooling $20 \mathrm{ml} / \mathrm{min}$ ).

\section{RMN 8-mm tip group}

All patients in the magnet group were ablated with the Navistar DS 8-mm catheter (Biosense Webster). The cavotricuspid isthmus was mapped with the aid of RMN (Stereotaxis, Niobe) and an electro-anatomical mapping system (CARTO, Biosense Webster). This was not used for activation mapping, but to tag the ablation line: the highest peak-to-peak bipolar electrograms were indicated on the map. Ablation was first directed to the highest potential on this map. If the ablation lesion did not result in bidirectional bwlock, the next largest atrial electrogram was subsequently targeted for ablation until bidirectional block was present. The magnetic navigation system was used to store the vectors into the system which allowed us to easily redirect the magnet catheter to the highest atrial electrogram, after the map of the cavotricuspid isthmus was made. The settings of the ablation were $60 \mathrm{~s}, 60 \mathrm{~W}, 50^{\circ} \mathrm{C}$. If we did not succeed in reaching bidirectional block within 15 applications; a switch to a manual guided cooled-tip catheter was allowed (Thermocool, Biosense Webster, settings cfr. supra).

\section{RMN irrigated tip group}

The same protocol was used as for the RMN 8-mm tip group, but with a Thermocool (Biosense Webster) with $60 \mathrm{~s}, 55 \mathrm{~W}, 48^{\circ} \mathrm{C}, 20$-ml cooling. 
Table 1 Patient characteristics

$\begin{array}{lcccc}\text { History of } & \text { Magnet } 8 \mathrm{~mm} & \text { Magnet irrigated tip } & \text { Conventional group } & \text { P value } \\ \text { Age } & 60 \pm 12 & 6 \pm 9 & 58 \pm 9 & \mathrm{~ns} \\ \text { Sex (F/M) } & 4 / 13 & 5 / 9 & 6 / 18 & \mathrm{~ns} \\ \text { AF } & 12 \% & 43 \% & 50 \% & \mathrm{n} \\ \text { Hypertension } & 12 \% & 14 \% & 25 \% & \mathrm{~ns} \\ \text { Ischaemic heart disease } & 6 \% & 7 \% & 8 \% & \mathrm{~ns} \\ \text { Valvular heart disease } & 6 \% & 14 \% & 4 \% & \mathrm{~ns} \\ \text { Pacemaker } & 12 \% & 0 \% & 4 \% & \mathrm{~ns} \\ \text { Peripheral vascular disease } & 0 \% & 0 \% & \mathrm{~ns}\end{array}$

\section{End points}

The primary end point was defined as the presence of bidirectional block within 15 applications. Secondary end points were the median number of applications needed to obtain acute success, the ablation time, procedural time, fluoroscopy time.

\section{Statistical analysis}

Continuous, normally distributed variables were compared with the ANOVA test and the Fisher exact test as post-hoc test. Continuous, not normally distributed variables were compared with the non-parametric Kruskal Wallis test. Not normally distributed variables are present as median \pm interquartile range. A $P$-value $<0.05$ was considered statistically significant.

\section{RESULTS}

\section{Patient characteristics}

The mean age of the patients was $59 \pm 10$ years (range 25-84 years) and 27\% was female. Thirty-six percent suffered from AF and 18\% from hypertension. Five patients had valvular heart disease, 3 patients had a pacemaker before ablation, 4 had known ischaemic heart disease, 1 patient peripheral vascular disease and 2 patients had a history of transient ischaemic attack (table 1).

\section{Procedural parameters}

There was no difference between the different groups concerning the primary end point. The proportion of patients who could successfully be treated within 15 applications was 59\% for the RMN 8-mm tip group, 64\% for the RMN irrigated tip group and $83 \%$ for the manual group $(P=0.19)$.
Patients treated in the magnet group (both 8-mm and irrigated tip) needed overall significantly more applications to obtain bidirectional block compared to the conventional group. The median number of applications was 10 in the RMN 8-mm tip group, 13 in the RMN irrigated tip group and 8 in the manual group $(P=0.03)$ (figure 1). The median application time was 570 seconds (RMN 8-mm tip group), 692 seconds (RMN irrigated tip group) and 465 seconds (manual group) $(P=0.03)$. Five or less applications were needed in $12 \%$ of the patients in the RMN 8-mm tip group, 7\% in the RMN irrigated tip group versus $17 \%$ in the conventional group $(P=0.69)$. In 5 patients from the RMN 8-mm tip group (29\%), the magnet catheter was switched to a conventional guided cooled-tip catheter. In 2 patients bidirectional block could be achieved after 1 extra application; in another 2 patients after 4 and 15 extra applications with the cooled-tip catheter, while in 1 patient we could not create bidirectional block at all. In 1 patient of the

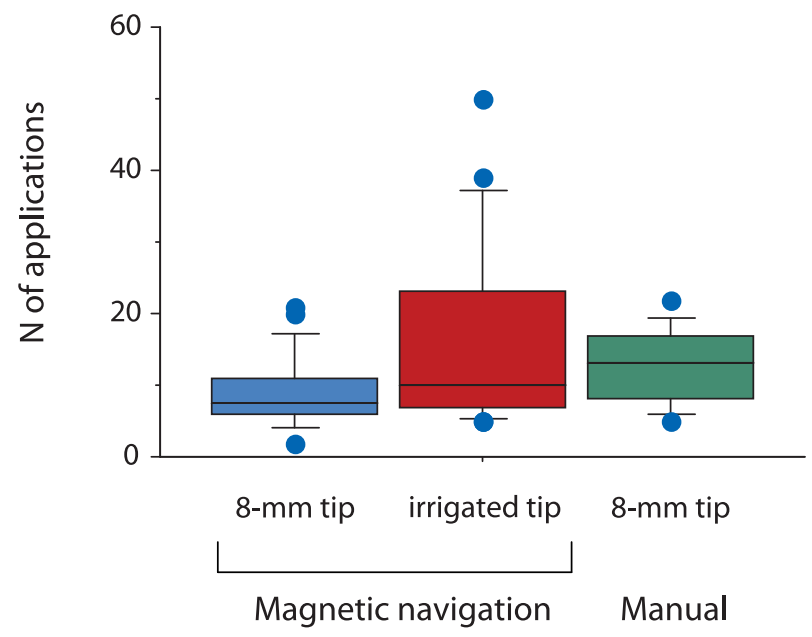

Fig. 1 Box-plot of the total number of applications needed to achieve bidirectional cavotricuspid isthmus block. 


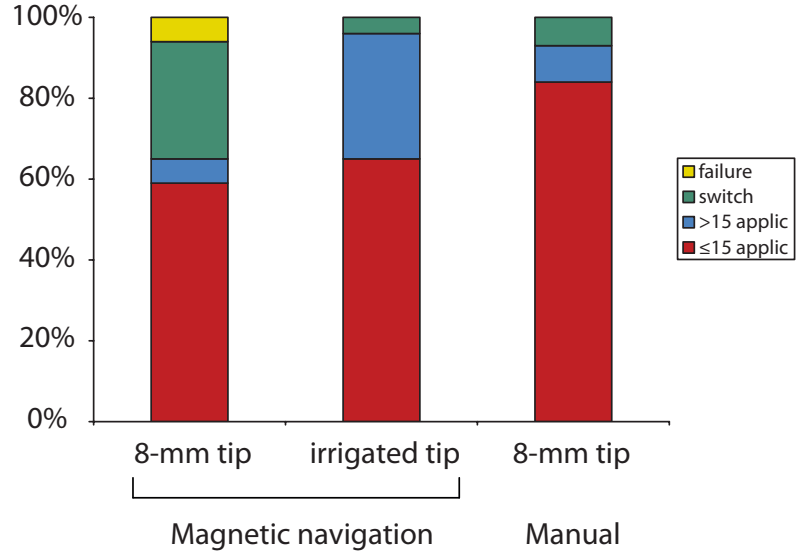

Fig. 2 Graph depicting the percentage of patients in whom bidirectional block could be achieved with $\leq 15$ applications, $>15$ applications, and after a switch to a manual navigated cooled-tip catheter and where no bidirectional block could be obtained.

RMN irrigated tip group (8\%) there was a switch to a conventional irrigated tip catheter. In this patient, 3 extra applications were needed to obtain complete isthmus block. In the manual group there was also 1 patient who was switched to an irrigated tip catheter (4\%). In this patient 8 extra applications were needed to obtain bidirectional block $(P=0.04)$ (figure 2$)$.

Procedural times were significantly longer in the magnet group compared to the conventional group: $148 \pm 44 \mathrm{~min}$ (RMN 8-mm tip group) $143 \pm 40 \mathrm{~min}$ (RMN irrigated tip group) versus $106 \pm 53 \mathrm{~min}$ (manual group) ( $P=0.03)$. There was no difference in fluoroscopy times: $23 \pm 12 \mathrm{~min}$ (RMN 8-mm tip group), $22 \pm 7 \mathrm{~min}$ (RMN irrigated tip group) and $20 \pm 11 \mathrm{~min}(P=0.62)$ (manual group) (table 2).

There was 1 adverse event, consisting of a false aneurysm in a patient of the RMN irrigated tip group.

\section{DISCUSSION}

The main finding of this study is that for the primary end point (success within 15 applications) there was no difference between the 3 catheters. Secondary end points showed that more applications were needed with RMN compared to the manual approach, and that procedural times were longer. A third finding is that the maximum voltage technique is feasible with RMN.

\section{Remote magnetic navigation}

RMN proved to be a feasible technique for ablation of the majority of arrhythmias and has shown superiority in some difficult arrhythmias, such as in congenital heart disease ${ }^{4}$. The major advantages are that it is a safe procedure since perforation with a floppy catheter is highly unlikely and that the amount of radiation for the operator substantially decreases, as he can operate the catheter remote from the patient ${ }^{9}$. However, it is not impossible that these measures compromise efficacy.

Recently the irrigated tip catheter for RMN was introduced, making the advantages of catheter tip cooling possible in the magnetic environment.

In atrial flutter we did not see a statistical difference in the primary end point (success within 15 applications) compared to manual ablation. Nevertheless, the results with the RMN 8-mm tip were disappointing. We needed more applications compared to a conventional approach, and we needed to switch 5 times to a manual irrigated tip catheter.

Our results with the RMN irrigated tip catheter were encouraging since fewer switches to a cooled-tip manual catheter were needed (5 versus 1 ). Newer technologies which are emerging, such as adjustable magnetic field strength, might help to achieve even higher acute success rates.

There are several possible explanations for these mixed results. First of all, there are many anatomical variants in the cavotricuspid isthmus: the isthmus can vary in length, there may be pouch-like recesses or a prominent Eustachian valve and the angle between the inferior vena cava and the isthmus have different degrees ${ }^{10}$. Troublesome ablation of atrial flutter is associated with a longer cavotricuspid length and with a rectangular angle between the isthmus and the vena cava

Table 2 Comparison of secondary end points between the different groups

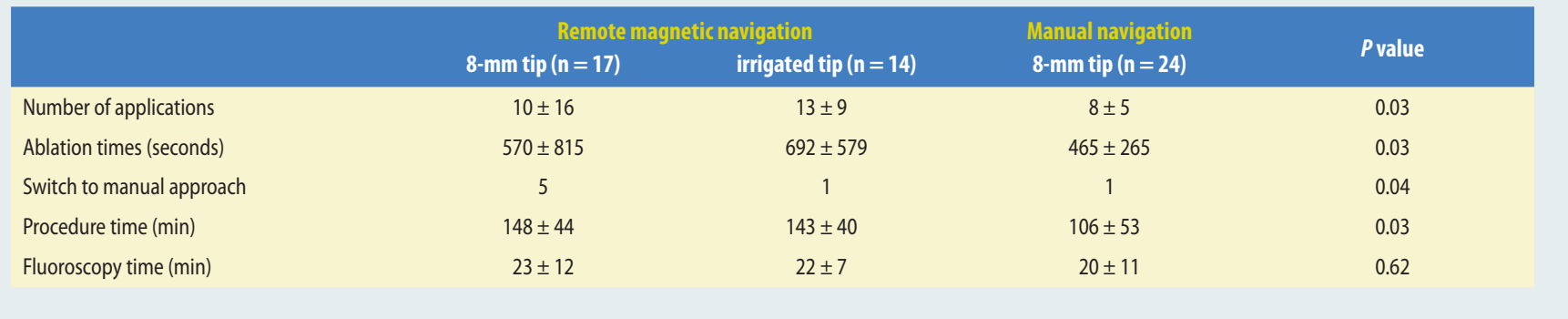


inferior ${ }^{11}$. The maximum tissue force which can be applied by RMN is less than the average and significantly less than the maximal force which can be applied using a standard manual catheter ${ }^{12}$. This can make it difficult to overcome some anatomical variations and to put the catheter parallel to the isthmus. When the catheter is placed more perpendicular than parallel to the tissue, the effective ablated contact area will decrease. When comparing lesions from magnetically steered catheters with standard manual catheters, the lesions from the former are more round or oval than elongated. The likelihood of seeing 'brush lesions'as is common with the standard manual catheter is substantially less with the magnetically steered catheter. However, these 'brush lesions' can actually be advantageous in the setting of flutter ablation ${ }^{13}$.

Particularly, when there is a rectangle between the isthmus and the vena cava inferior, it is sometimes difficult to have good wall contact at the transition of the isthmus and the vena cava inferior. Especially, an 8-mm magnetic catheter will have disadvantages both when the cavotricuspid isthmus is irregular, and when the angle with the vena cava inferior is sharp.

The use of a RMN 8-mm tip catheter has been described in other studies. Vollmann et al. found that acute success rate was reduced, more applications to obtain isthmus block were necessary, with longer procedural times and a reduced long-term success ${ }^{6}$. Similar results have been presented with acute success ranging from $54 \%$ till $89 \%{ }^{14,15}$. Only in one study a success rate of $100 \%$ was reported in a group of 24 patients, a success which was maintained after 6 months of follow-up in $92 \%$ of the patients ${ }^{11,16}$. The use of the irrigated tip catheter could improve these results, since it is easier to titrate the energy, and as deeper lesions are formed.

The difference in procedural time is partly explained by the use of the electro-anatomical mapping system (CARTO) in the RMN group, even when activation was analysed. Since the EP recording system cannot measure voltages, we needed to use this system to exactly measure the highest peak-to-peak bipolar atrial electrograms. The combination of CARTO and RMN needs additional time for set-up and registering both systems and can explain a large part of the extra time needed in the RMN groups.

\section{Maximum voltage technique}

The approach using the maximum voltage technique led to a significant decrease in the number of applications compared to former approaches. In this series, we needed a median of 8 applications to achieve bidirectional block (conventional manual group). This is far less then the average of 23 applications when we created continuous lines as published before ${ }^{17}$. The procedural time decreased as well in the historical control group from $170 \pm 48 \mathrm{~min}$ to $106 \pm 53 \mathrm{~min}$, fluoroscopy time from $29 \pm 15$ min till $20 \pm 11 \mathrm{~min}^{17}$. The aid of magnetic navigation, however, did not add to a further decrease in the number of applications and fluoroscopy times as initially expected. This might be due to the fact that several of these procedures were done in a training programme, and because the set-up of the electromagnetic system required additional time in the RMN, which was shared with the intervention group.

\section{LIMITATIONS}

Whether a patient was appointed to the magnet group or the conventional group was based on the availability of the Stereotaxis room, and not on a randomization system. We did not perform angiography of the right atrium in order to assess the characteristics of the cavotricuspid isthmus. However, we have no reason to presume that the distribution of the different anatomical variants is different between the 3 groups. Unfortunately, no comparison was made with a conventional irrigated tip group.

\section{CONCLUSIONS}

The use of magnetic navigation for the ablation of atrial flutter with the maximum voltage technique is feasible but not superior to a manual approach. Overall, more applications are needed and procedure times are longer (unfortunately influenced by other equipment in the two groups). Nevertheless, RMN and irrigated tip technology, required fewer switching to manual catheters, which is promising. Hopefully, further improvements in the catheter design and the magnetic navigation system, such as an adjustable magnetic force, can make this safe approach also more performing.

\section{ACKNOWLEDGEMENTS}

Wim Anné has been supported by a clinical fellowship of the European Society of Cardiology.

\section{CONFLICTS OF INTEREST}

Tamas Szili-Torok received a moderate consultancy fee from Stereotaxis Inc. Luc Jordaens received a research grant from Stereotaxis Inc. The other authors have no conflicts of interest to declare. 


\section{REFERENCES}

1. Blomstrom-Lundqvist $C$, Scheinman MM, Aliot EM, Alpert JS, Calkins H, Camm AJ, Campbell WB, Haines DE, Kuck KH, Lerman BB, Miller DD, Shaeffer CW, Jr., Stevenson WG, Tomaselli GF, Antman EM, Smith SC, Jr. Faxon DP, Fuster V, Gibbons RJ, Gregoratos G, Hiratzka LF, Hunt SA, Jacobs AK, Russell RO, Jr., Priori SG, Blanc JJ, Budaj A, Burgos EF, Cowie M, Deckers JW, Garcia MA, Klein WW, Lekakis J, Lindahl B, Mazzotta G, Morais JC, Oto A, Smiseth O, Trappe HJ. ACC/AHA/ESC guidelines for the management of patients with supraventricular arrhythmias--executive summary: a report of the American College of Cardiology/American Heart Association Task Force on Practice Guidelines and the European Society of Cardiology Committee for Practice Guidelines (Writing Committee to Develop Guidelines for the Management of Patients With Supraventricular Arrhythmias). Circulation 2003; 108(15): 1871-1909.

2. Pappone $C$, Vicedomini $G$, Manguso $F$, Gugliotta F, Mazzone P, Gulletta S, Sora N, Sala S, Marzi A, Augello G, Livolsi L, Santagostino A, Santinelli V. Robotic magnetic navigation for atrial fibrillation ablation. J Am Coll Cardiol 2006; 47(7): 1390-1400.

3. Thornton AS, Janse $P$, Theuns DA, Scholten MF, Jordaens LJ. Magnetic navigation in AV nodal re-entrant tachycardia study: early results of ablation with one- and three-magnet catheters. Europace 2006; 8(4): 225-30.

4. Schwagten B, Cuypers J, Szili-Torok T. The magnetic navigation system allows avoidance of puncturing a baffle during ablation of a postincisional macroreentrant tachycardia. Cardiol Young 2009: 19(2): 216-9.

5. Aryana A, d'Avila A, Heist EK, Mela T, Singh JP, Ruskin JN, Reddy VY. Remote magnetic navigation to guide endocardial and epicardial catheter mapping of scar-related ventricular tachycardia Circulation 2007; 115(10): 1191-200.

6. Vollmann D, Luthje L, Seegers J, Hasenfuss G, Zabel M. Remote magnetic catheter navigation for cavotricuspid isthmus ablation in patients with common-type atrial flutter. Circ Arrhythm Electrophysiol 2009; 2(6): 603-10.

7. Cabrera JA, Sanchez-Quintana D, Farre J, Rubio JM, Ho SY. The inferior right atrial isthmus: further architectural insights for current and coming ablation technologies. J Cardiovasc Electrophysiol 2005; 16(4): 402-8.

8. Redfearn DP, Skanes AC, Gula LJ Krahn AD, Yee R, Klein GJ. Cavotricuspid isthmus conduction is dependent on underlying anatomic bundle architecture: observations using a maximum voltage-guided ablation technique. J Cardiovasc Electrophysiol 2006; 17(8): 832-8.

9. Thornton AS, Maximo Rivero-Ayerza M Jordaens LJ. Magnetic assisted navigation in electrophysiology and cardiac resynchronisation: a review. Indian Pacing Electrophysiol J 2006; 6(4): 202-13.

10. Heidbuchel $H$, Willems $R$, van Rensburg $H_{\text {, }}$ Adams J, Ector H, Van de Werf F. Right atrial angiographic evaluation of the posterior isthmus: relevance for ablation of typical atrial flutter. Circulation 2000; 101(18): 2178-84

11. Kim AM, Turakhia M, Lu J, Badhwar N, Lee BK, Lee RJ, Marcus GM, Tseng ZH, Scheinman M, Olgin JE. Impact of remote magnetic catheter navigation on ablation fluoroscopy and procedure time. Pacing Clin Electrophysiol 2008; 31(11): 1399-404.

12. Faddis MN, Blume W, Finney J, Hall A, Rauch J, Sell J, Bae KT, Talcott M,
Lindsay B. Novel, magnetically guided catheter for endocardial mapping and radiofrequency catheter ablation. Circulation 2002; 106(23): 2980-5.

13. Thornton AS, De Castro CA, van Deel E, van Beusekom HM, Jordaens L. An in vivo comparison of radiofrequency cardiac lesions formed by standard and magnetically steered $4 \mathrm{~mm}$ tip catheters. Neth Heart J 2010; 18(2): 66-71.

14. Koektuerk B, Chun J, Schmidt B, Metzner A, Tilz R, Ernst S, Koester I, Ouyang F, Kuck KH. Remote controlled catheter ablation of typical atrial flutter: A preliminary comparison of the magnetic $8-\mathrm{mm}$ tip catheter versus the novel $3.5-\mathrm{mm}$ irrigated tip magnetic catheter (abstract) Europace 2008; 10 supplement 1: i176.

15. Preminger $M$, Kamath $G$, Sichrovsky $T$, Arshad A, Mittal S, Steinberg J. Early experience with remote magnetic navigation ablation of cavotricuspid isthmus. Potential limitations (abstract). Heart Rhythm 2009; 6 issue supplement 1: S61.

16. Huo Y, Hindricks G, Piorkowski C, Bollmann A, Wetzel U, Sommer P, Gaspar T, Kottkamp H, Arya A. Six-month follow-up of isthmus-dependent right atrial flutter ablation using a remote magnetic catheter navigation system: a case-control study. Acta Cardiol 2010; 65(3): 279-83.

17. Thornton AS, Janse $P$, Alings $M$, Scholten MF, Mekel JM, Miltenburg M, Jessurun $E$, Jordaens L. Acute success and short-term follow-up of catheter ablation of isthmus-dependent atrial flutter; a comparison of $8 \mathrm{~mm}$ tip radiofrequency and cryothermy catheters. J Interv Card Electrophysiol 2008; 21(3): 241-8. 